\title{
BACKWARD ERROR ANALYSIS OF CYCLIC REDUCTION FOR THE SOLUTION OF TRIDIAGONAL SYSTEMS
}

\author{
PIERLUIGI AMODIO AND FRANCESCA MAZZIA
}

\begin{abstract}
Tridiagonal systems play a fundamental role in matrix computation. In particular, in recent years parallel algorithms for the solution of tridiagonal systems have been developed. Among these, the cyclic reduction algorithm is particularly interesting. Here the stability of the cyclic reduction method is studied under the assumption of diagonal dominance. A backward error analysis is made, yielding a representation of the error matrix for the factorization and for the solution of the linear system. The results are compared with those for $L U$ factorization.
\end{abstract}

\section{INTRODUCTION}

Tridiagonal matrices arise in a large variety of applications. It is known that for the solution of linear systems, the $L U$ factorization is the best scalar algorithm. Since it is not efficient for parallel computation, many algorithms which may be easily parallelized have been proposed [11, 13, 15]. Among many others, the cyclic reduction algorithm appears to be the most interesting [1, 2, $3,4,9,10,14]$. As a result, an increasing number of papers have been written in the last ten years which consider cyclic reduction.

So far, no backward error analysis has been made. The main result about cyclic reduction is given by Heller [6], concerning block tridiagonal systems. Heller shows that norms of the off-diagonal blocks (relative to the diagonal blocks) decrease quadratically with each reduction. This is useful when an approximate solution is desired, as in a preconditioner based on cyclic reduction.

A backward error analysis is carried out in the following sections. We use a block representation of the algorithm, which provides a simpler way to analyze the propagation of the error.

In the case of diagonal dominance it is well known that, for the $L U$ factorization, the backward error in the solution obtained in floating-point arithmetic may be bounded from above by a quantity independent of the dimension of the matrix $[8,16]$. Therefore, the algorithm is numerically stable. The results of our backward error analysis for the cyclic reduction algorithm show that, for diagonally dominant matrices, the backward error of the computed solution is

Received by the editor October 30, 1991 and, in revised form, September 25, 1992.

1991 Mathematics Subject Classification. Primary 65F05, 65G05, 15 A23.

Key words and phrases. Tridiagonal linear systems, cyclic reduction, backward error analysis.

Work performed within the activities of the project 'Matematica computazionale' supported by MURST 40\% and by "Progetto finalizzato Sistemi Informatici e Calcolo Parallelo. Sottoprogetto: Calcolo Scientifico per Grandi Sistemi” of C.N.R. 
bounded by a factor which depends on the logarithm of the dimension of the linear system. This is a satisfactory growth rate for error propagation, but in fact it is a pessimistic upper bound. In numerical tests we show that a more accurate upper bound may be computed which is comparable to that of $L U$ factorization.

In $\S 2$ a brief description of the algorithm is presented and a block representation for the factorization of the coefficient matrix is proposed. In $\S \S 3-4$ the backward error analysis for the factorization and for the solution of the linear system is presented. This analysis takes into account the special structure of the matrices involved in the propagation of the error.

\section{BLOCK REPRESENTATION OF THE CYCLIC REDUCTION ALGORITHM}

Consider the following system of linear equations:

$$
M \mathbf{x}=\mathbf{f},
$$

where the coefficient matrix $M$ is tridiagonal,

$$
M=\left(\begin{array}{cccc}
a_{1} & b_{1} & & \\
c_{2} & a_{2} & \ddots & \\
& \ddots & \ddots & b_{n-1} \\
& & c_{n} & a_{n}
\end{array}\right) .
$$

We derive the cyclic reduction algorithm by considering a block factorization of $M$. By means of an odd-even permutation matrix $P_{1}$ (which transforms the sequence $1, \ldots, n$ into the sequence $1,3,5, \ldots, 2,4,6, \ldots)$ the matrix $M$ is expressed as a $2 \times 2$ block matrix

$$
P_{1} M P_{1}^{T}=\left(\begin{array}{ll}
A_{1} & T_{1} \\
S_{1} & B_{1}
\end{array}\right)
$$

with $A_{1}$ and $B_{1}$ diagonal matrices

$$
A_{1}=\left(\begin{array}{ccc}
a_{1} & & \\
& a_{3} & \\
& & \ddots
\end{array}\right), \quad B_{1}=\left(\begin{array}{lll}
a_{2} & & \\
& a_{4} & \\
& & \ddots .
\end{array}\right)
$$

and $S_{1}$ and $T_{1}$ bidiagonal (not necessarily square) matrices

$$
S_{1}=\left(\begin{array}{cccc}
c_{2} & b_{2} & & \\
& c_{4} & b_{4} & \\
& & \ddots & \ddots
\end{array}\right), \quad T_{1}=\left(\begin{array}{ccc}
b_{1} & & \\
c_{3} & b_{3} & \\
& \ddots & \ddots
\end{array}\right) .
$$

The permuted matrix $(2.3)$ is factored as

$$
P_{1} M P_{1}^{T}=L_{1} D_{1} U_{1},
$$

where $L_{1}$ and $U_{1}$ are block triangular

$$
L_{1}=\left(\begin{array}{cc}
I & \\
S_{1} A_{1}^{-1} & I
\end{array}\right), \quad U_{1}=\left(\begin{array}{cc}
A_{1} & T_{1} \\
& I
\end{array}\right)
$$


and $D_{1}$ is block diagonal

$$
D_{1}=\left(\begin{array}{ll}
I & \\
& M_{1}
\end{array}\right)
$$

with a tridiagonal block $M_{1}=B_{1}-S_{1} A_{1}^{-1} T_{1}$ of dimension $\lfloor n / 2\rfloor \times\lfloor n / 2\rfloor\left(M_{1}\right.$ is the Schur complement of $A_{1}$ in $P_{1} M P_{1}^{T}$ ).

The same procedure is applied to $M_{1}$ (or equivalently to $D_{1}$, leaving unchanged the top left block). That is, we consider a $\lfloor n / 2\rfloor \times\lfloor n / 2\rfloor$ odd-even permutation matrix $Q_{2}$ and

$$
P_{2}=\left(\begin{array}{ll}
I & \\
& Q_{2}
\end{array}\right)
$$

and carry out the factorization

$$
D_{1}=P_{2}^{T}\left(\begin{array}{ccc}
I & & \\
& A_{2} & T_{2} \\
& S_{2} & B_{2}
\end{array}\right) P_{2}=P_{2}^{T} L_{2} D_{2} U_{2} P_{2}
$$

in which $D_{2}$ is block diagonal and contains a tridiagonal sub-block $M_{2}$ of dimension $\lfloor n / 4\rfloor \times\lfloor n / 4\rfloor$. By iterating this process of reduction, after $j$ steps the matrix

$$
D_{j-1}=\left(\begin{array}{ll}
I & \\
& M_{j-1}
\end{array}\right)
$$

(where $D_{0}=M$ ) is factored in the form

$$
D_{j-1}=P_{j}^{T} L_{j} D_{j} U_{j} P_{j}
$$

where

$$
\begin{gathered}
L_{j}=\left(\begin{array}{ccc}
I & & \\
& I & \\
& S_{j} A_{j}^{-1} & I
\end{array}\right), \quad U_{j}=\left(\begin{array}{ccc}
I & & \\
& A_{j} & T_{j} \\
& & I
\end{array}\right), \\
D_{j}=\left(\begin{array}{lll}
I & & \\
& I & \\
& & M_{j}
\end{array}\right) .
\end{gathered}
$$

This new block decomposition is obtained so that the blocks on the main diagonal are square of dimension respectively $n-\left\lfloor n / 2^{j-1}\right\rfloor$ on the first row, $\left\lfloor n / 2^{j-1}\right\rfloor-\left\lfloor n / 2^{j}\right\rfloor$ on the second, $\left\lfloor n / 2^{j}\right\rfloor$ on the third. The reduction process stops after $k=\left\lfloor\log _{2} n\right\rfloor$ steps when the block $M_{k}$ is $1 \times 1$ and $D_{k}$ is diagonal.

The following summarizes the factorization of the matrix $M$ :

$$
M=P_{1}^{T} L_{1} P_{2}^{T} L_{2} \cdots P_{k}^{T} L_{k} D_{k} U_{k} P_{k} \cdots U_{2} P_{2} U_{1} P_{1} .
$$

Consider now a block representation of the cyclic reduction algorithm for the solution of the problem (2.1) that will be useful for the stability analysis.

\section{Algorithm 1. Let}

a) $M_{0}=M$ be a tridiagonal matrix of order $n, \mathbf{y}_{0}=\mathbf{f}$;

b) $Q_{j}$, for $j=1, \ldots, k=\left\lfloor\log _{2} n\right\rfloor$, be an odd-even permutation matrix of 
order $j$ such that

$$
\left(\begin{array}{cc}
A_{j} & T_{j} \\
S_{j} & B_{j}
\end{array}\right)=Q_{j} M_{j-1} Q_{j}^{T} \quad \text { and } \quad\left(\begin{array}{c}
\mathbf{y}_{j-1}^{o} \\
\mathbf{y}_{j-1}^{e}
\end{array}\right)=Q_{j} \mathbf{y}_{j-1} .
$$

The following algorithm computes the solution $\mathbf{x}_{0}$ of $M \mathbf{x}=\mathbf{f}$ by cyclic reduction:

$$
\begin{aligned}
& \text { for } j=1, k \\
& \quad V_{j}=S_{j} A_{j}^{-1} \\
& \quad M_{j}=B_{j}-V_{j} T_{j} \\
& \quad \mathbf{y}_{j}=\mathbf{y}_{j-1}^{e}-V_{j} \mathbf{y}_{j-1}^{o} \\
& \text { end } \\
& \mathbf{x}_{k}=M_{k}^{-1} \mathbf{y}_{k} \\
& \text { for } j=k, 1,-1 \\
& \quad \mathbf{x}_{j-1}=Q_{j}^{T}\left(\begin{array}{c}
A_{j}^{-1}\left(\mathbf{y}_{j-1}^{o}-T_{j} \mathbf{x}_{j}\right) \\
\mathbf{x}_{j}
\end{array}\right) \\
& \text { end }
\end{aligned}
$$

Denote by $a_{i}, b_{i}, c_{i}$ respectively the nonzero elements on the main diagonal, the upper and the lower off-diagonal of $M_{j-1}$, and by $a_{i}^{\prime}, b_{i}^{\prime}, c_{i}^{\prime}$ the respective elements of $M_{j}$ (when it is not confusing, we always simplify the notation and let $a_{i}, b_{i}, c_{i}$ be the generic elements $a_{i}^{(r)}, b_{i}^{(r)}, c_{i}^{(r)}$ of $\left.M_{r}\right)$. Let further $s_{i}$ and $t_{i}$ denote the generic elements on the two nonzero diagonals of $V_{j}$. Then, if $m=\left\lfloor n / 2^{j}\right\rfloor$, the elements of the matrices $V_{j}$ and $M_{j}$ in Algorithm 1 are computed as follows:

$$
\begin{array}{ll}
\text { for } & i=1, m \\
& s_{i}=c_{2 i} / a_{2 i-1} \\
& t_{i}=b_{2 i} / a_{2 i+1} \quad\left(t_{m}=0 \text { if }\left\lfloor n / 2^{j-1}\right\rfloor \text { is odd }\right) \\
& a_{i}^{\prime}=a_{2 i}-s_{i} b_{2 i-1}-t_{i} c_{2 i+1} \\
& b_{i}^{\prime}=-t_{i} b_{2 i+1} \quad\left(b_{m}^{\prime}=0\right) \\
& c_{i}^{\prime}=-s_{i} c_{2 i-1} \quad\left(c_{1}^{\prime}=0\right) \\
\text { end } &
\end{array}
$$

while the algorithms for the $j$ th step in the solution of the two linear systems are:

$$
\begin{array}{ll}
\text { for } & i=1, m \\
& y_{i}^{(j)}=y_{2 i}^{(j-1)}-s_{i} y_{2 i-1}^{(j-1)}-t_{i} y_{2 i+1}^{(j-1)} \\
\text { end } &
\end{array}
$$

and

$$
\text { for } \begin{aligned}
& i=1, m \\
& \\
& x_{2 i-1}^{(j-1)}=\left(y_{2 i-1}^{(j-1)}-c_{2 i-1} x_{i-1}^{(j)}-b_{2 i-1} x_{i}^{(j)}\right) / a_{2 i-1} \\
& x_{2 i}^{(j-1)}=x_{i}^{(j)}
\end{aligned}
$$

end

if $\left\lfloor n / 2^{j-1}\right\rfloor$ is odd $x_{2 m+1}^{(j-1)}=\left(y_{2 m+1}^{(j-1)}-c_{2 m+1} x_{m}^{(j)}\right) / a_{2 m+1}$

The factorization of the coefficient matrix $M$ requires $8 n$ operations while the solution of the linear systems requires $9 n$ operations. The number of operations is twice the number of operations of the $L U$ factorization algorithm, but this algorithm is easier to parallelize. 
We introduce now the following convention:

$$
\prod_{j=1}^{i} A_{j}=A_{1} A_{2} \cdots A_{i}
$$

and we recall an important characterization of the factorization, also contained in [6], that will be used in the next sections.

Theorem 1. The cyclic reduction factorization for the matrix $M$,

$$
M=\prod_{i=1}^{k} P_{i}^{T} L_{i} D_{k} \prod_{i=1}^{k} U_{k-i+1} P_{k-i+1},
$$

may be expressed as the $L U$ factorization of the permuted matrix

$$
\prod_{i=1}^{k} P_{i} M \prod_{i=1}^{k} P_{k-i+1}^{T} .
$$

The matrices $M_{i}$ (with $i$ varying from 1 to $k=\left\lfloor\log _{2} n\right\rfloor$ ) enjoy special properties which are useful for studying the stability of the factorization. The two following theorems concern diagonally dominant matrices (see [17] for the proofs).

Theorem 2. If $M_{0}=M$ is diagonally dominant by rows (by columns), then all the matrices $M_{i}$ are diagonally dominant by rows (by columns).

Moreover, it has been proved that if $M$ is strictly diagonally dominant, then the ratio between each element on the off-diagonal and the corresponding one on the main diagonal of $M_{i}$ decreases quadratically as $i$ varies from 1 to $\left\lfloor\log _{2} n\right\rfloor$ [6].

Theorem 3. If $M$ is diagonally dominant by rows or by columns, then for each matrix $M_{i}$ there holds

$$
\left\|M_{i}\right\|_{\infty} \leq\|M\|_{\infty}
$$

\section{BACKWARD ERROR ANALYSIS}

Assume that computations are carried out in floating-point arithmetic following the model

$$
\begin{aligned}
\mathrm{fl}(x \text { op } y)=(x \text { op } y)\left(1+u_{1}\right) \text { and } \quad \mathrm{fl}(x \text { op } y)=\frac{x \text { op } y}{1+u_{2}}, \\
\left|u_{i}\right| \leq u, \text { for } i=1,2,
\end{aligned}
$$

where $u$ is the unit roundoff and op $\in\{+,-, *, /\}$.

The implementation of the cyclic reduction algorithm on a computer for the solution of the problem (2.1) gives, instead of the exact solution $\mathbf{x}$, an approximate solution $\tilde{\mathbf{x}}$. In the following we obtain an upper bound for the infinity norm of the matrix $\Delta M$ such that $\tilde{\mathbf{x}}$ is the exact solution of the perturbed problem

$$
(M+\Delta M) \tilde{\mathbf{x}}=\mathbf{f} .
$$

when $M$ is diagonally dominant. 
In the backward error analysis we introduce a matrix $\delta M$ containing the error due to the factorization. Let

$$
\mathrm{L}=\prod_{j=1}^{k} P_{j}^{T} \tilde{L}_{j} \quad \text { and } \quad \mathrm{U}=\tilde{D}_{k} \prod_{j=1}^{k} \tilde{U}_{k-j+1} P_{k-j+1}
$$

where $\tilde{D}_{k}, \tilde{L}_{j}, \tilde{U}_{j}$ are the computed matrices $D_{k}, L_{j}$, and $U_{j}$ of (2.5). Then

$$
\delta M=\mathrm{LU}-M
$$

Moreover, we consider two further error matrices $\delta \mathrm{L}$ and $\delta \mathrm{U}$ arising in the numerical solution of the two triangular systems with matrices $L$ and $U$, so that $\tilde{\mathbf{x}}$ is the exact solution of the system

$$
(\mathrm{L}+\delta \mathrm{L})(\mathrm{U}+\delta \mathrm{U}) \tilde{\mathbf{x}}=\mathbf{f} .
$$

In first-order approximation we obtain (for any compatible norm)

$$
\|\Delta M\| \leq\|\delta M+\delta \mathrm{L} \mathrm{U}+\mathrm{L} \delta \mathrm{U}\|,
$$

while the relative error of the solution may be bounded by

$$
\frac{\|\tilde{\mathbf{x}}-\mathbf{x}\|}{\|\tilde{\mathbf{x}}\|} \leq\left\|M^{-1}\right\|\|\Delta M\| \text {. }
$$

To obtain the error matrices $\delta M, \delta \mathrm{L}$, and $\delta \mathrm{U}$, we use an approach which permits exploiting the structure of sparsity of the error matrix.

The factorization of the matrix $M$ is obtained by means of the $k$ steps of factorization previously considered. At each step $j$, a new block-diagonal matrix $D_{j}$, see (2.4), (with the last block being tridiagonal of dimension $\left\lfloor n / 2^{j}\right\rfloor$ ) is obtained from the matrix $D_{j-1}$.

The relations between $D_{j}$ and $D_{j-1}$ are expressed by means of the following matrix difference equation:

$$
\left\{\begin{array}{l}
D_{0}=M \\
D_{j}=L_{j}^{-1} P_{j} D_{j-1} P_{j}^{T} U_{j}^{-1}
\end{array}\right.
$$

In floating-point arithmetic, we have

$$
\tilde{D}_{j}=\tilde{L}_{j}^{-1} P_{j} \tilde{D}_{j-1} P_{j}^{T} \tilde{U}_{j}^{-1}+\delta D_{j}
$$

where $\delta D_{j}$ contains the errors committed in the $j$ th step of the factorization. In order to evaluate the error matrix $\delta D_{j}$, we consider the floating-point operations (see the description of Algorithm 1):

$$
\left(\tilde{V}_{j}\right)_{r, i}= \begin{cases}\tilde{c}_{2 i} / \tilde{a}_{2 i-1}\left(1+u_{1}\right)=\tilde{s}_{i} & \text { if } r=i \\ \tilde{b}_{2 i} / \tilde{a}_{2 i+1}\left(1+u_{2}\right)=\tilde{t}_{i} & \text { if } r=i-1\end{cases}
$$


and

$$
\left(\tilde{M}_{j}\right)_{r, i}=\left\{\begin{array}{c}
-\tilde{t}_{i} \tilde{b}_{2 i+1}\left(1+u_{3}\right)=\tilde{b}_{i}^{\prime} \quad \text { if } r=i-1, \\
\tilde{a}_{2 i}\left(1+u_{7}\right)-\tilde{s}_{i} \tilde{b}_{2 i-1}\left(1+u_{4}+u_{6}+u_{7}\right) \\
-\tilde{t}_{i} \tilde{c}_{2 i+1}\left(1+u_{5}+u_{6}+u_{7}\right)=\tilde{a}_{i}^{\prime} \quad \text { if } r=i, \\
-\tilde{s}_{i} \tilde{c}_{2 i-1}\left(1+u_{8}\right)=\tilde{c}_{i}^{\prime} \quad \text { if } r=i+1,
\end{array}\right.
$$

where $\left|u_{i}\right| \leq u$, or in block form:

$$
\tilde{V}_{j}=\tilde{S}_{j} \tilde{A}_{j}^{-1}+\delta V_{j}, \quad \tilde{M}_{j}=\tilde{B}_{j}-\tilde{V}_{j} \tilde{T}_{j}+\delta M_{j},
$$

where $\delta V_{j}$ is lower bidiagonal and $\delta M_{j}$ is tridiagonal. There follows

$$
\left|\delta V_{j}\right| \leq\left|\tilde{S}_{j} \tilde{A}_{j}^{-1}\right| u, \quad\left|\delta M_{j}\right| \leq\left|\tilde{B}_{j}-\tilde{V}_{j} \tilde{T}_{j}\right| u+2 \operatorname{diag}\left(\left|\tilde{V}_{j}\right|\left|\tilde{T}_{j}\right|\right) u,
$$

where $|A|$ denotes the matrix of the absolute values of the elements of $A$, $\operatorname{diag}(A)$ the diagonal matrix containing the main diagonal of $A$, and inequalities are understood componentwise.

Let

$$
\tilde{L}_{j}=\left(\begin{array}{ccc}
I & & \\
& I & \\
& \tilde{V}_{j} & I
\end{array}\right), \quad \tilde{D}_{j}=\left(\begin{array}{ccc}
I & & \\
& I & \\
& & \tilde{M}_{j}
\end{array}\right), \quad \tilde{U}_{j}=\left(\begin{array}{ccc}
I & & \\
& \tilde{A}_{j} & \tilde{T}_{j} \\
& & I
\end{array}\right)
$$

then from (3.4) one gets

$$
\delta D_{j}=\left(\begin{array}{ccc}
0 & & \\
& 0 & \\
& -\delta V_{j} & \delta V_{j} \tilde{T}_{j}-\delta M_{j}
\end{array}\right) .
$$

Theorem 4. The error matrix $\delta M$ defined in (3.1) satisfies

$$
\delta M=\sum_{j=1}^{k} \prod_{i=1}^{j} P_{i}^{T} H_{j}^{F} \prod_{i=1}^{j} P_{j-i+1}
$$

where

$$
H_{j}^{F}=\left(\begin{array}{ccc}
0 & & \\
& 0 & \\
& \delta V_{j} \tilde{A}_{j} & \delta M_{j}
\end{array}\right)
$$

Proof. To show how the error propagates, we define

$$
\begin{gathered}
\epsilon_{0}=\tilde{D}_{0}-D_{0}=\tilde{M}_{0}-M=0 \\
\epsilon_{j}=\tilde{D}_{j}-D_{j}=\tilde{L}_{j}^{-1} P_{j} \tilde{D}_{j-1} P_{j}^{T} \tilde{U}_{j}^{-1}+\delta D_{j}-L_{j}^{-1} P_{j} D_{j-1} P_{j}^{T} U_{j}^{-1} .
\end{gathered}
$$

By adding and subtracting the expressions

$$
\tilde{L}_{j}^{-1} P_{j} D_{j-1} P_{j}^{T} \tilde{U}_{j}^{-1} \text { and } L_{j}^{-1} P_{j} D_{j-1} P_{j}^{T} \tilde{U}_{j}^{-1}
$$


we obtain

$$
\begin{aligned}
\epsilon_{j}= & \tilde{L}_{j}^{-1} P_{j} \epsilon_{j-1} P_{j}^{T} \tilde{U}_{j}^{-1}+\delta D_{j}+\left(\tilde{L}_{j}^{-1}-L_{j}^{-1}\right) P_{j} D_{j-1} P_{j}^{T} \tilde{U}_{j}^{-1} \\
& +L_{j}^{-1} P_{j} D_{j-1} P_{j}^{T}\left(\tilde{U}_{j}^{-1}-U_{j}^{-1}\right)=\tilde{L}_{j}^{-1} P_{j} \epsilon_{j-1} P_{j}^{T} \tilde{U}_{j}^{-1}+Z_{j}
\end{aligned}
$$

where

$$
Z_{j}=\left(\begin{array}{ccc}
0 & A_{j} \tilde{A}_{j}^{-1}-I & T_{j}-A_{j} \tilde{A}_{j}^{-1} \tilde{T}_{j} \\
& \delta V_{j}+\left(V_{j}-\tilde{V}_{j}\right) A_{j} \tilde{A}_{j}^{-1} & \delta M_{j}-\delta V_{j} \tilde{T}_{j}+\left(V_{j}-\tilde{V}_{j}\right)\left(T_{j}-A_{j} \tilde{A}_{j}^{-1} \tilde{T}_{j}\right)
\end{array}\right) .
$$

Solving the difference equation in (3.8) yields

$$
\epsilon_{k}=\sum_{j=1}^{k} \prod_{i=1}^{k-j}\left(P_{k-i+1}^{T} \tilde{L}_{k-i+1}\right)^{-1} Z_{j} \prod_{i=j+1}^{k}\left(\tilde{U}_{i} P_{i}\right)^{-1}
$$

For $j=1, \ldots, k$, let $\mathrm{L}_{j}=\prod_{i=1}^{j} P_{i}^{T} \tilde{L}_{i}$ and $\mathrm{U}_{j}=\prod_{i=1}^{j} \tilde{U}_{j-i+1} P_{j-i+1}\left(\mathrm{~L}_{k}=\right.$ $\mathrm{L}$ and $\left.\mathrm{U}_{k}=\mathrm{U}\right)$. We multiply both members of the equation from the left by $\mathrm{L}_{k}$ and from the right by $\mathrm{U}_{k}$ to obtain

$$
\mathrm{L}_{k}\left(\tilde{D}_{k}-D_{k}\right) \mathrm{U}_{k}=\sum_{j=1}^{k} \mathrm{~L}_{j} Z_{j} \mathrm{U}_{j}
$$

and hence

$$
\mathrm{L}_{k} \tilde{D}_{k} \mathrm{U}_{k}=\mathrm{L}_{k} D_{k} \mathrm{U}_{k}+\sum_{j=1}^{k} \mathrm{~L}_{j} Z_{j} \mathrm{U}_{j}=\mathrm{L}_{k}\left(D_{k}+Z_{k}\right) \mathrm{U}_{k}+\sum_{j=1}^{k-1} \mathrm{~L}_{j} Z_{j} \mathrm{U}_{j}
$$

As to the first term on the far right of (3.9), we have

$$
\mathrm{L}_{k-1} P_{k}^{T} \tilde{L}_{k}\left(D_{k}+Z_{k}\right) \tilde{U}_{k} P_{k} \mathrm{U}_{k-1}=\mathrm{L}_{k-1} D_{k-1} \mathrm{U}_{k-1}+\mathrm{L}_{k-1} P_{k}^{T} H_{k}^{F} P_{k} \mathrm{U}_{k-1} \text {. }
$$

By iterating on $\mathrm{L}_{k-1} D_{k-1} \mathrm{U}_{k-1}$ and on the second term on the far right of (3.9) it follows that

$$
\delta M=\mathrm{L}_{k} \tilde{D}_{k} \mathrm{U}_{k}-M=\sum_{j=1}^{k} \mathrm{~L}_{j-1} P_{j}^{T} H_{j}^{F} P_{j} \mathrm{U}_{j-1}
$$

The matrix

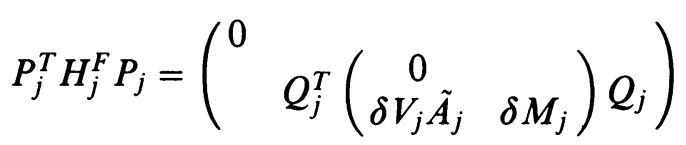

has only a $\left\lfloor n / 2^{j-1}\right\rfloor \times\left\lfloor n / 2^{j-1}\right\rfloor$ block on the main diagonal different from zero. By what was said in the previous section, the matrices $\mathrm{L}_{j-1}$ and $\mathrm{U}_{j-1}$ are respectively lower and upper triangular, and they have the identity matrix in place of the nonnull block of (3.11). The result follows by simplifying the expression in (3.10).

In order to obtain an upper bound for the norm of $\delta M$, we consider the following 
Theorem 5. If $M$ is diagonally dominant, then the matrices $H_{j}^{F}$ in (3.6) satisfy

$$
\left\|H_{j}^{F}\right\|_{\infty} \leq\left\|\tilde{M}_{j}\right\|_{\infty} u+2\left\|\tilde{M}_{j-1}\right\|_{\infty} u \leq 3\|M\|_{\infty} u,
$$

and for the error matrix we have

$$
\|\delta M\|_{\infty} \leq 3 k\|M\|_{\infty} u
$$

Proof. See the Appendix.

\section{STABILITY OF THE SOlUTION OF THE TRIANGULAR SYSTEMS}

Because of roundoff errors, instead of the exact solution of the lower triangular system, we obtain an approximate solution $\tilde{\mathbf{y}}$ which is the exact solution of a problem

$$
(\mathrm{L}+\delta \mathrm{L}) \tilde{\mathbf{y}}=\mathbf{f} .
$$

To calculate $\delta \mathrm{L}$, we introduce, for each $L_{i}$, an error matrix $\delta L_{i}$. From Algorithm 1 we see that the error at the step $j$ can be obtained from

$$
\tilde{y}_{i}^{(j)}=\frac{\tilde{y}_{2 i}^{(j-1)}-\left(\tilde{s}_{i} \tilde{y}_{2 i-1}^{(j-1)}\left(1+u_{1}+u_{3}\right)+\tilde{t}_{i} \tilde{y}_{2 i+1}^{(j-1)}\left(1+u_{2}+u_{3}\right)\right.}{1+u_{4}}, \quad\left|u_{i}\right| \leq u,
$$

or, if written in block form, from

$$
\tilde{\mathbf{y}}_{j}=\left(I+\delta \zeta_{j}\right)^{-1}\left(\tilde{\mathbf{y}}_{j-1}^{e}-\left(\tilde{V}_{j}+\delta \tilde{V}_{j}\right) \tilde{\mathbf{y}}_{j-1}^{o}\right)
$$

where

$$
\left|\delta \tilde{V}_{j}\right| \leq 2\left|\tilde{V}_{j}\right| u, \quad\left|\delta \zeta_{j}\right| \leq I u .
$$

Therefore, the error matrix at the step $j$ is

$$
\delta L_{j}=\left(\begin{array}{ccc}
0 & & \\
& 0 & \\
& \delta \tilde{V}_{j} & \delta \zeta_{j}
\end{array}\right) .
$$

Our aim is to find a suitable bound for the norm in (3.2). For this, we first consider the product $\delta \mathrm{LU}$.

Theorem 6. The product $\delta \mathrm{LU}$ introduced in (3.2) satisfies

$$
\delta \mathrm{LU}=\sum_{j=1}^{k} \prod_{i=1}^{j} P_{i}^{T} H_{j}^{L} \prod_{i=1}^{j} P_{j-i+1}
$$

where

$$
H_{j}^{L}=\left(\begin{array}{ccc}
0 & & \\
& 0 & \\
& \delta \tilde{V}_{j} \tilde{A}_{j} & \delta \tilde{V}_{j} \tilde{T}_{j}+\delta \zeta_{j} \tilde{M}_{j}
\end{array}\right) .
$$


Proof. In first-order approximation, we have

$$
\prod_{j=1}^{k} P_{j}^{T}\left(\tilde{L}_{j}+\delta L_{j}\right)=\prod_{j=1}^{k} P_{j}^{T} \tilde{L}_{j}+\sum_{j=1}^{k} \mathrm{~L}_{j-1} P_{j}^{T} \delta L_{j} \prod_{i=j+1}^{k} P_{i}^{T} \tilde{L}_{i}=\mathrm{L}+\delta \mathrm{L} .
$$

Then

$$
\begin{aligned}
\delta \mathrm{L} \mathrm{U} & =\sum_{j=1}^{k} \mathrm{~L}_{j-1} P_{j}^{T} \delta L_{j} \prod_{i=j+1}^{k} P_{i}^{T} \tilde{L}_{i} \tilde{D}_{k} \prod_{i=1}^{k} \tilde{U}_{k-i+1} P_{k-i+1} \\
& =\sum_{j=1}^{k} \mathrm{~L}_{j-1} P_{j}^{T} \delta L_{j} \tilde{D}_{j} \mathrm{U}_{j} .
\end{aligned}
$$

The structures of $\mathrm{L}_{j-1}$ and $\mathrm{U}_{j}$ allow us to simplify the expression (4.6) and to obtain the following final expression for $\delta \mathrm{LU}$ :

$$
\delta \mathrm{LU}=\sum_{j=1}^{k} \prod_{i=1}^{j} P_{i}^{T} \delta L_{j} \tilde{D}_{j} \tilde{U}_{j} \prod_{i=1}^{j} P_{j-i+1}
$$

From this, the assertion follows.

The next theorem establishes an upper bound for the infinity norm of $\delta \mathrm{LU}$. Theorem 7. If $M$ is diagonally dominant, then the matrices $H_{j}^{L}$ in (4.5) satisfy

$$
\left\|H_{j}^{L}\right\|_{\infty} \leq 2\left(\left\|\tilde{M}_{j}\right\|_{\infty}+\left\|\tilde{M}_{j-1}\right\|_{\infty}\right) u \leq 4\|M\|_{\infty} u
$$

and

$$
\|\delta \mathrm{LU}\|_{\infty} \leq 4 k\|M\|_{\infty} u \text {. }
$$

Proof. See the Appendix.

Similar results can be obtained for the solution of the upper triangular system. The following theorems hold.

Theorem 8. The product $\mathrm{L} \delta \mathrm{U}$ introduced in (3.2) satisfies

$$
\mathrm{L} \delta \mathrm{U}=\sum_{j=1}^{k} \prod_{i=1}^{j} P_{i}^{T} H_{j}^{U} \prod_{i=1}^{j} P_{k-i+1}
$$

where

$$
\begin{aligned}
& H_{j}^{U}=\left(\begin{array}{ccc}
0 & & \\
& \delta \tilde{A}_{j} & \delta \tilde{T}_{j} \\
& \tilde{V}_{j} \delta \tilde{A}_{j} & \tilde{V}_{j} \delta \tilde{T}_{j}
\end{array}\right) \quad \text { for } j<k, \\
& H_{k}^{U}=\left(\begin{array}{ccc}
0 & & \\
& \delta \tilde{A}_{k} & \delta \tilde{T}_{k} \\
& \tilde{V}_{k} \delta \tilde{A}_{k} & \tilde{V}_{k} \delta \tilde{T}_{k}+\delta \tilde{M}_{k}
\end{array}\right),
\end{aligned}
$$


and

$$
\left|\delta \tilde{M}_{k}\right| \leq\left|\tilde{M}_{k}\right| u, \quad\left|\delta \tilde{A}_{j}\right| \leq 2\left|\tilde{A}_{j}\right| u, \quad\left|\delta \tilde{T}_{j}\right| \leq 2\left|\tilde{T}_{j}\right| u \text {. }
$$

Theorem 9. If $M$ is diagonally dominant, then the matrices $H_{j}^{U}$ in (4.9) satisfy

and

$$
\left\|H_{j}^{U}\right\|_{\infty} \leq 2\left\|\tilde{M}_{j-1}\right\|_{\infty} u+\left\|\tilde{M}_{j}\right\|_{\infty} u \leq 3\|M\|_{\infty} u
$$

$$
\|\mathrm{L} \delta \mathrm{U}\|_{\infty} \leq 3 k\|M\|_{\infty} u \text {. }
$$

The next theorem summarizes the results for the backward and forward error analyses made in this and in the previous section.

Theorem 10. If the tridiagonal $n \times n$ matrix $M$ is diagonally dominant by rows or by columns then, by using the cyclic reduction for solving $M \mathbf{x}=\mathbf{f}$, the computed solution $\tilde{\mathbf{x}}$ satisfies, in first-order approximation,

$$
(M+\Delta M) \tilde{\mathbf{x}}=\mathbf{f}, \quad\|\Delta M\|_{\infty} \leq 10 \log _{2} n\|M\|_{\infty} u
$$

and

$$
\frac{\|\tilde{\mathbf{x}}-\mathbf{x}\|_{\infty}}{\|\tilde{\mathbf{x}}\|_{\infty}} \leq 10 \log _{2} n \kappa(M) u
$$

where $\kappa(M)$ is the condition number of the matrix $M$ in the infinity norm.

Proof. The backward error derives from (3.12), (4.7), and (4.10). The forward error is obtained from (3.3).

\section{NUMERICAL EXPERIMENTS}

We have carried out numerical experiments to compare our estimates of the relative error in the cyclic reduction $(C R)$ algorithm with known estimates in the $L U$ factorization. For the $L U$ factorization we have used in our comparision the following upper bound (see $[8,16]$ ):

$$
\frac{\|\tilde{\mathbf{x}}-\mathbf{x}\|_{\infty}}{\|\tilde{\mathbf{x}}\|_{\infty}} \leq 8 \kappa(M) u
$$

Numerical experiments indicate that the upper bound (4.11) is too pessimistic. In order to improve it, we have computed the following sharper bound for the norm of the error matrix, using (3.5), (4.4), and (4.8):

$$
\|\Delta M\|_{\infty} \leq\left\|\sum_{j=1}^{k} \prod_{i=1}^{j} P_{i}^{T}\left(\left|H_{j}^{F}\right|+\left|H_{j}^{L}\right|+\left|H_{j}^{U}\right|\right) \prod_{i=1}^{j} P_{j-i+1}\right\|_{\infty} u .
$$

We then have from (3.3) the following upper bound:

$$
\frac{\|\tilde{\mathbf{x}}-\mathbf{x}\|_{\infty}}{\|\tilde{\mathbf{x}}\|_{\infty}} \leq \kappa(M) \frac{\|\Delta M\|_{\infty}}{\|M\|_{\infty}},
$$

where $\|\Delta M\|_{\infty}$ can be estimated by (5.2). 


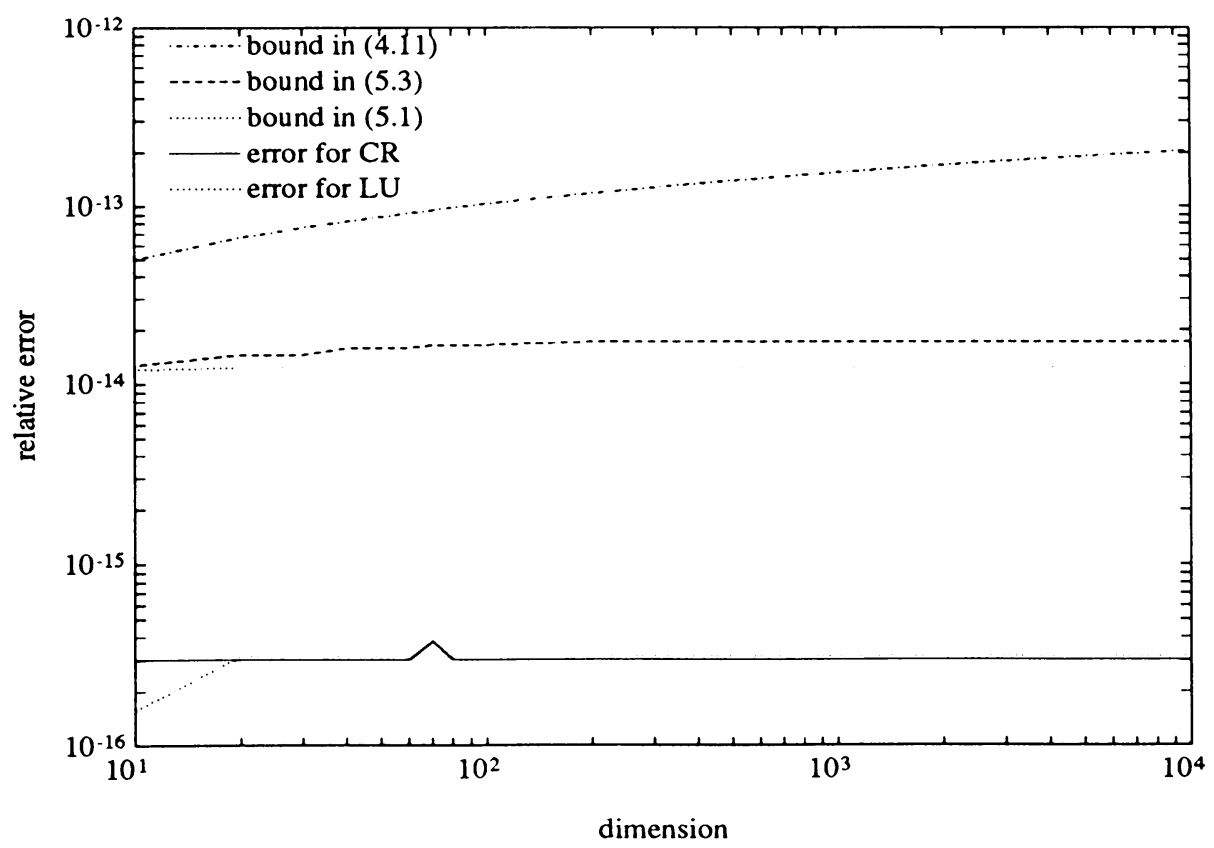

FIGURE 1. Upper bounds and relative errors for the test problem 1

We report on four numerical examples, all having different coefficient matrices. The right-hand sides $f$ were always chosen in order to obtain

$$
\mathbf{x}=1.4142 \cdot(2,-1,2,-1, \ldots 2,-1)^{T}
$$

as the solution of problem (2.1). We used the algorithm in [7] to compute the norm of the inverse of the coefficient matrix.

Test problem 1 (see Figure 1). Consider the diagonally dominant Toeplitz matrix

$$
M=\left(\begin{array}{cccc}
4 & -1 & & \\
-2 & 4 & \ddots & \\
& \ddots & \ddots & -1 \\
& & -2 & 4
\end{array}\right)_{n \times n},
$$

with condition number $\kappa(M) \leq 7$. The solution of $(2.1)$ by the $C R$ algorithm and by the $L U$ factorization is obtained to within machine precision. Therefore, the upper bounds are excessively large. In particular, there is no error growth corresponding to the term $\log _{2} n$ in (4.11).

Test problem 2 (see Figure 2). Consider a symmetric weakly diagonally dominant Toeplitz matrix

$$
M=\left(\begin{array}{cccc}
5 & -2.5 & & \\
-2.5 & 5 & \ddots & \\
& \ddots & \ddots & -2.5 \\
& & -2.5 & 5
\end{array}\right)_{n \times n}
$$




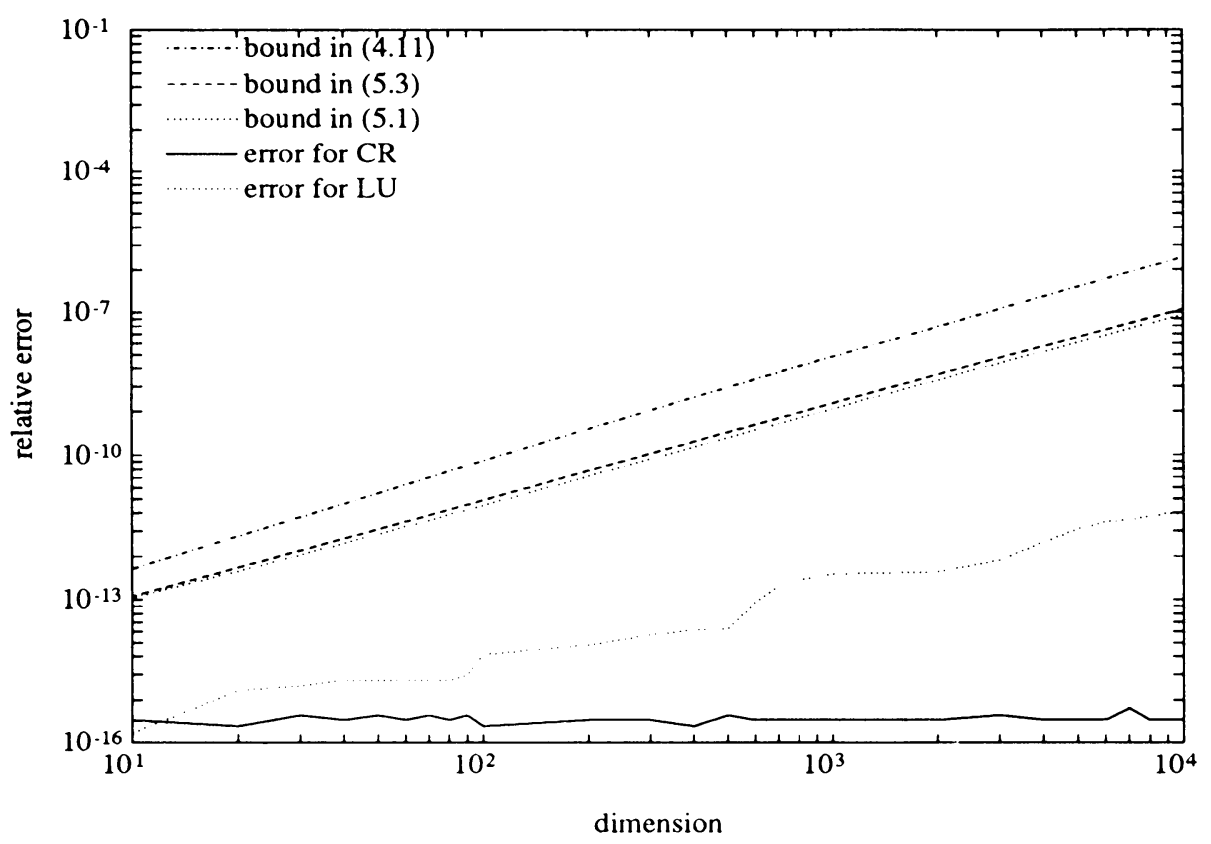

FIGURE 2. Upper bounds and relative errors for the test problem 2

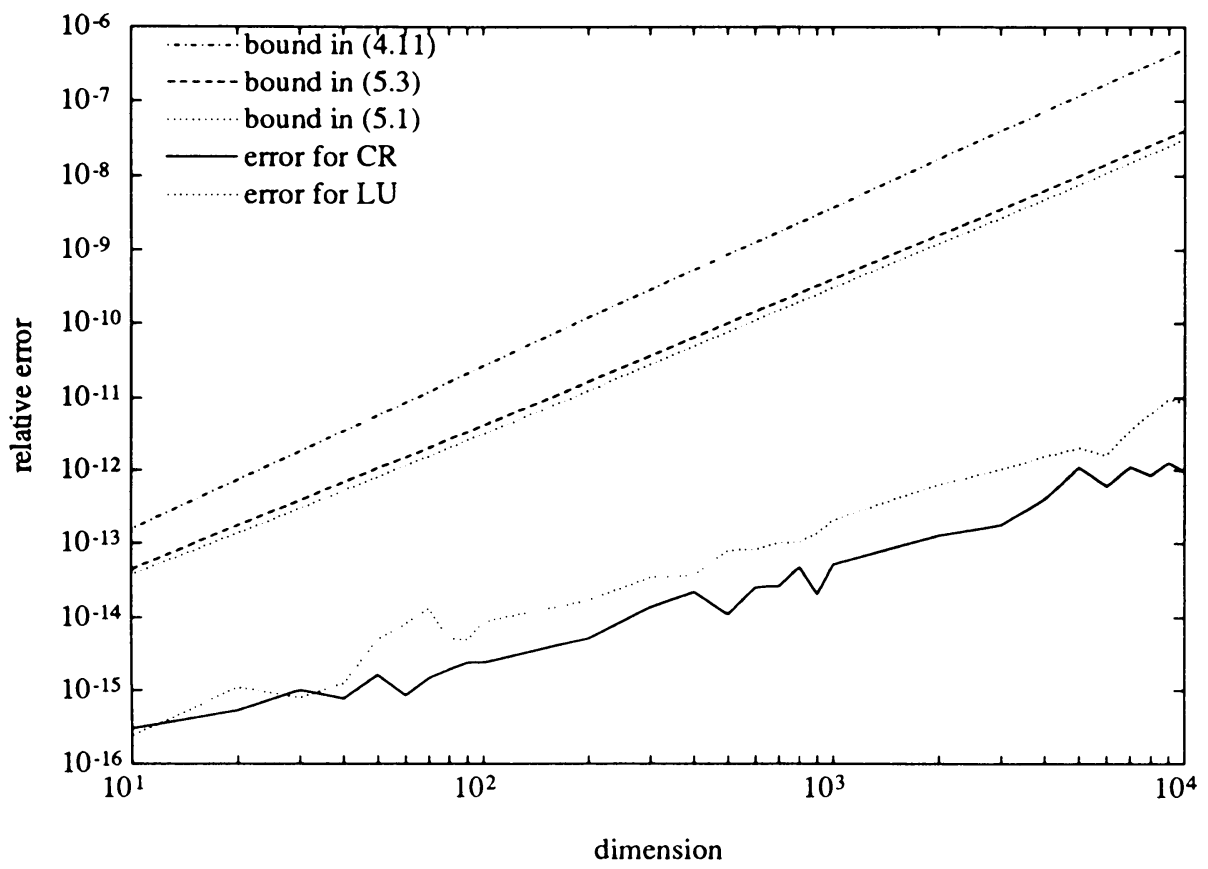

FIGURE 3. Upper bounds and relative errors for the test problem 3

For matrices in this class, operations of the $C R$ algorithm are performed exactly. Therefore the relative error is proportional to the machine precision. The condition number and the relative error for the $L U$ factorization algorithm grow as $O\left(n^{2}\right)$.

Test problem 3 (see Figure 3 ). Consider a weakly diagonally dominant matrix $M$, of order $n$, defined by (see (2.2)) 


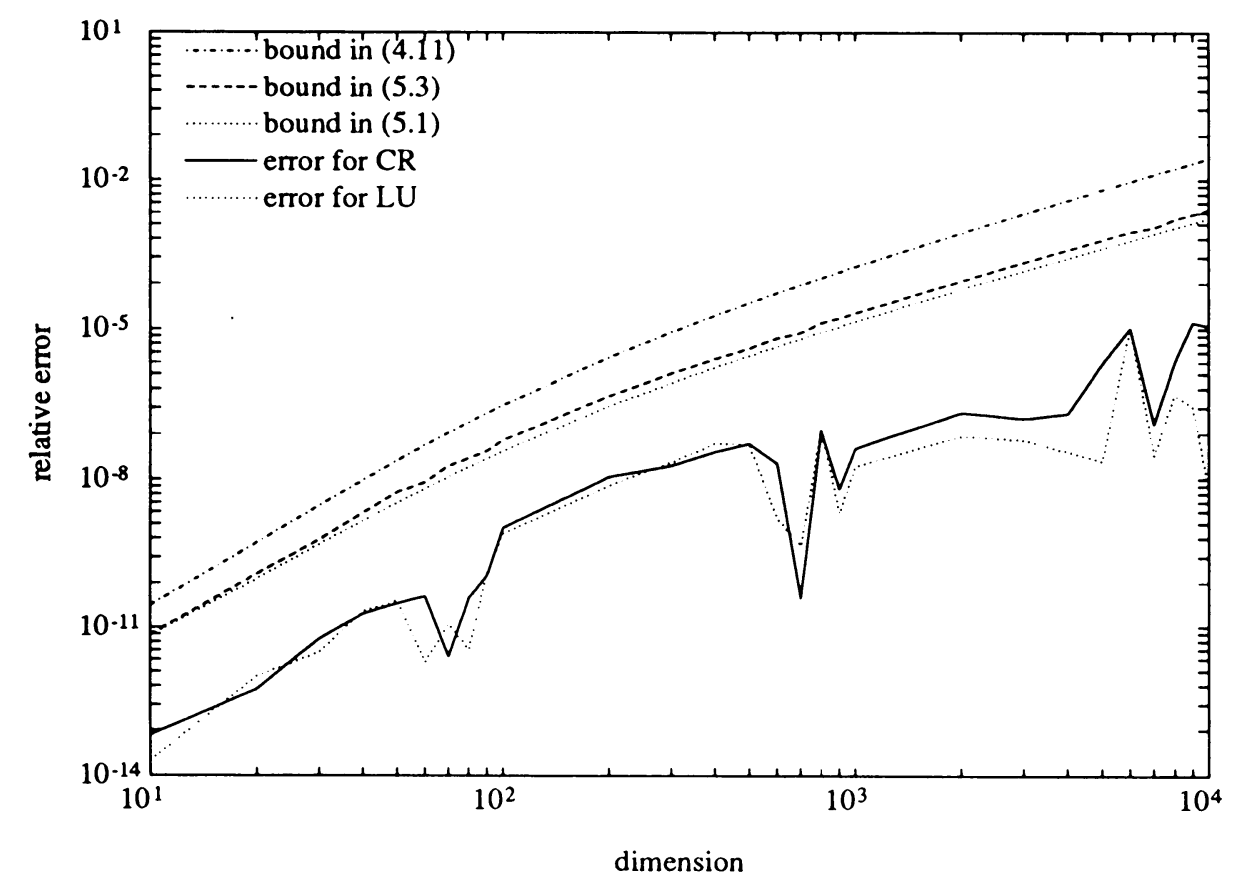

FIGURE 4. Upper bounds and relative errors for the test problem 4

$$
a_{i}=1, \quad b_{i}=-\frac{2 \epsilon+(1-i \cdot h) h}{4 \epsilon}, \quad c_{i}=-\frac{2 \epsilon-(1-i \cdot h) h}{4 \epsilon},
$$

where $h=2 /(n+1)$. This matrix occurs in the discretization of a singular perturbation problem by finite differences (see [12]). We chose $\epsilon=0.1$ in order to have diagonal dominance. Its condition number is $O\left(n^{2}\right)$.

Test problem 4 (see Figure 4). An ill-conditioned problem $\left(\kappa(M)=1.64 \cdot 10^{9}\right.$ for $n=500$ ) derived from the discretization of another singular perturbation problem by finite differences given in [5, 8]. The $n \times n$ coefficient matrix $M$ is defined by

$$
\begin{aligned}
& c_{i}= \begin{cases}-\frac{\epsilon}{h^{2}} & \text { if } 1 \leq i \leq\left\lfloor\frac{n+1}{2}\right\rfloor, \\
-\frac{\epsilon}{h^{2}}+\frac{0.5-i \cdot h}{h} & \text { if }\left\lfloor\frac{n+1}{2}\right\rfloor+1 \leq i \leq n,\end{cases} \\
& b_{i}= \begin{cases}-\frac{\epsilon}{h^{2}}-\frac{0.5-i \cdot h}{h} & \text { if } 1 \leq i \leq\left\lfloor\frac{n+1}{2}\right\rfloor, \\
-\frac{\epsilon}{h^{2}} & \text { if }\left\lfloor\frac{n+1}{2}\right\rfloor+1 \leq i \leq n,\end{cases} \\
& a_{i}=-\left(b_{i}+c_{i}\right),
\end{aligned}
$$

where $h=1 /(n+1)$ and $\epsilon=0.009$. The numbers $c_{1}$ and $b_{n}$ do not occur in the matrix $M$, but are only introduced to define $a_{1}$ and $a_{n}$. The matrix $M$ is a nonsingular, row diagonally dominant M-matrix, but it becomes singular for $\epsilon \rightarrow 0$. 
We conclude that the cyclic reduction algorithm is stable for tridiagonal diagonally dominant matrices. From our experiments we observe that the dependence on $\log _{2} n$ is pessimistic and does not describe the actual behavior of the error (the upper bound in (5.3) has the same behavior as that in (5.1)).

\section{APPENDIX}

Proof of Theorem 5. The matrices $\delta V_{j}$ and $\delta M_{j}$ have the following structure:

$$
\delta V_{j}=\left(\begin{array}{cccc}
\delta s_{1}^{(j)} & \delta t_{1}^{(j)} & & \\
& \delta s_{2}^{(j)} & \delta t_{2}^{(j)} & \\
& & \ddots & \ddots
\end{array}\right) \quad \text { and } \delta M_{j}=\left(\begin{array}{ccc}
\delta a_{1}^{(j)} & \delta b_{1}^{(j)} & \\
\delta c_{2}^{(j)} & \delta a_{2}^{(j)} & \ddots \\
& \ddots & \ddots
\end{array}\right) \text {, }
$$

where

$$
\begin{aligned}
& \left|\delta s_{i}^{(j)}\right| \leq\left|\tilde{c}_{2 i}^{(j-1)} / \tilde{a}_{2 i-1}^{(j-1)}\right| u, \quad\left|\delta t_{i}^{(j)}\right| \leq\left|\tilde{b}_{2 i}^{(j-1)} / \tilde{a}_{2 i+1}^{(j-1)}\right| u, \\
& \left|\delta a_{i}^{(j)}\right| \leq\left|\tilde{a}_{i}^{(j)}\right| u+2\left|\tilde{s}_{i}^{(j)} \tilde{b}_{2 i-1}^{(j-1)}\right| u+2\left|\tilde{t}_{i}^{(j)} \tilde{c}_{2 i+1}^{(j-1)}\right| u, \\
& \left|\delta b_{i}^{(j)}\right| \leq\left|\tilde{t}_{i}^{(j)} \tilde{b}_{2 i+1}^{(j-1)}\right| u, \quad\left|\delta c_{i}^{(j)}\right| \leq\left|\tilde{s}_{i}^{(j)} \tilde{c}_{2 i-1}^{(j-1)}\right| u .
\end{aligned}
$$

From the hypothesis of diagonal dominance we have

$$
\left|\tilde{s}_{i}^{(j)} \tilde{b}_{2 i-1}^{(j-1)}\right|+\left|\tilde{t}_{i}^{(j)} \tilde{c}_{2 i+1}^{(j-1)}\right| \leq\left|\tilde{c}_{2 i}^{(j-1)}\right|+\left|\tilde{b}_{2 i}^{(j-1)}\right| \leq\left|\tilde{a}_{2 i}^{(j-1)}\right|
$$

and therefore

$$
\begin{aligned}
\left\|H_{j}^{F}\right\|_{\infty} & \leq \max _{i}\left(\left|\delta a_{i}^{(j)}\right|+\left|\delta b_{i}^{(j)}\right|+\left|\delta c_{i}^{(j)}\right|+\left|\delta s_{i}^{(j)} a_{2 i-1}^{(j-1)}\right|+\left|\delta t_{i}^{(j)} a_{2 i+1}^{(j-1)}\right|\right) \\
& \leq \max _{i}\left(\left|\tilde{a}_{i}^{(j)}\right|+2\left|\tilde{a}_{2 i}^{(j-1)}\right|+\left|\tilde{b}_{i}^{(j)}\right|+\left|\tilde{c}_{i}^{(j)}\right|+\left|\tilde{b}_{2 i}^{(j-1)}\right|+\left|\tilde{c}_{2 i}^{(j-1)}\right|\right) u \\
& \leq\left\|\tilde{M}_{j}\right\|_{\infty} u+2\left\|\tilde{M}_{j-1}\right\|_{\infty} u .
\end{aligned}
$$

The assertion now follows from Theorem 3 and (3.7).

Proof of Theorem 7. From (4.1) and (4.2) we obtain

$$
\delta \tilde{V}_{j}=\left(\begin{array}{cccc}
\delta \tilde{s}_{1}^{(j)} & \delta \tilde{t}_{1}^{(j)} & & \\
& \delta \tilde{s}_{2}^{(j)} & \delta \tilde{t}_{2}^{(j)} & \\
& & \ddots & \ddots
\end{array}\right)
$$

where

$$
\left|\delta \tilde{s}_{i}^{(j)}\right| \leq 2\left|\tilde{c}_{2 i}^{(j-1)} / \tilde{a}_{2 i-1}^{(j-1)}\right| u, \quad\left|\delta \tilde{t}_{i}^{(j)}\right| \leq 2\left|\tilde{b}_{2 i}^{(j-1)} / \tilde{a}_{2 i+1}^{(j-1)}\right| u .
$$

Moreover, from (4.3) we have

$$
\left|\delta \zeta_{j} \tilde{M}_{j}\right| \leq\left|\tilde{M}_{j}\right| u
$$


From the diagonal dominance we conclude

$$
\begin{aligned}
\left\|H_{j}^{L}\right\|_{\infty} \leq & \max _{i}\left(2\left(\left|\tilde{s}_{i}^{(j)} \tilde{b}_{2 i-1}^{(j-1)}\right|+\left|\tilde{t}_{i}^{(j)} \tilde{c}_{2 i+1}^{(j-1)}\right|+\left|\tilde{s}_{i}^{(j)} \tilde{c}_{2 i-1}^{(j-1)}\right|+\left|\tilde{t}_{i}^{(j)} \tilde{b}_{2 i+1}^{(j-1)}\right|\right)\right. \\
& \left.\quad+\left|\tilde{a}_{i}^{(j)}\right|+\left|\tilde{b}_{i}^{(j)}\right|+\left|\tilde{c}_{i}^{(j)}\right|+2\left(\left|\tilde{s}_{i}^{(j)} \tilde{a}_{2 i-1}^{(j-1)}\right|+\left|\tilde{t}_{i}^{(j)} \tilde{a}_{2 i+1}^{(j-1)}\right|\right)\right) u \\
\leq & 2 \max _{i}\left(\left|\tilde{a}_{2 i}^{(j-1)}\right|+\left|\tilde{c}_{i}^{(j)}\right|+\left|\tilde{b}_{i}^{(j)}\right|+\left|\tilde{a}_{i}^{(j)}\right|+\left|\tilde{b}_{2 i}^{(j-1)}\right|+\left|\tilde{c}_{2 i}^{(j-1)}\right|\right) u \\
\leq & 2\left(\left\|\tilde{M}_{j}\right\|_{\infty}+\left\|\tilde{M}_{j-1}\right\|_{\infty}\right) u .
\end{aligned}
$$

The assertion now follows from Theorem 3 and (3.7).

\section{ACKNOWLEDGMENTS}

We are very grateful to Professor Donato Trigiante for his helpful comments and suggestions. Moreover, we are very indebted to Professor W. Gautschi and to the referee for suggestions which have improved our paper.

\section{BIBLIOGRAPHY}

1. P. Amodio, Optimized cyclic reduction for the solution of linear tridiagonal systems on parallel computers, Comput. Math Appl. 26 (1993), 45-53.

2. P. Amodio and L. Brugnano, Parallel factorizations and parallel solvers for tridiagonal linear systems, Linear Algebra Appl. 172 (1992), 347-364.

3. P. Amodio, L. Brugnano, and T. Politi, Parallel factorizations for tridiagonal matrices, SIAM J. Numer. Anal. 30 (1993), 813-823.

4. B. L. Buzbee, G. H. Golub, and C. W. Nielson, On direct methods for solving Poisson's equations, SIAM J. Numer. Anal. 7 (1970), 627-656.

5. F. W. Dorr, An example of ill-conditioning in the numerical solution of singular perturbation problems, Math. Comp. 25 (1971), 271-283.

6. D. Heller, Some aspects of the Cyclic Reduction Algorithm for block tridiagonal linear systems, SIAM J. Numer. Anal. 13 (1976), 484-496.

7. N. J. Higham, Efficient algorithms for computing the condition number of a tridiagonal matrix, SIAM J. Sci. Statist. Comput. 7 (1986), 150-165.

8. __ Bounding the error in Gaussian elimination for tridiagonal systems, SIAM J. Matrix Anal. Appl. 11 (1990), 521-530.

9. S. L. Johnsson, Solving tridiagonal systems on ensemble architectures, SIAM J. Sci. Statist. Comput. 8 (1987), 354-392.

10. D. Kershaw, Solution of single tridiagonal linear systems and vectorization of the ICCG algorithm on the CRAY 1, Parallel Computations (G. Rodrigue, ed.), Academic Press, New York, 1982, pp. 85-99.

11. J. J. Lambiotte, R. G. Voigt, The solution of tridiagonal linear systems on the CDC Star-100 computer, ACM Trans. Math. Software 1 (1975), 308-329.

12. F. Mazzia and D. Trigiante, Numerical methods for second order singular perturbation problems, Comput. Math. Appl. 23 (1992), 81-89.

13. J. M. Ortega, Introduction to parallel and vector solution of linear systems, Plenum Press, New York, 1988.

14. H. S. Stone, An efficient parallel algorithm for the solution of a tridiagonal linear system of equations, J. Assoc. Comput. Mach. 20 (1973), 27-38. 
15. H. A. Van der Vorst, Large tridiagonal and block tridiagonal linear systems on vector and parallel computers, Parallel Comput. 5 (1987), 45-54.

16. J. H. Wilkinson, The algebraic eigenvalue problem, Oxford Univ. Press, Oxford, 1965.

17. _ Error analysis of direct methods of matrix inversion, J. Assoc. Comput. Mach. 8 (1961), 281-330.

Dipartimento di Matematica, Universita di Bari, Via Orabona, 4, I-70125 Bari, Italy

E-mail address: 001105700vm.csata.it 